\title{
Influence of Polytypism on the Electronic Structure of CdSe/CdS and CdSe/CdSe Core/Shell Nanocrystals
}

\author{
Carlos Segarra, Fernando Rajadell, Juan I. Climente, and Josep Planelles* \\ Departament de Química Física i Analítica, Universitat Jaume I, E-12080, Castelló de la \\ Plana, Spain \\ E-mail: josep.planelles@uji.es
}




\begin{abstract}
We address theoretically differences and similarities on the electronic structure of CdSe/CdS dot-in-dot nanocrystals (NCs) with wurtzite/wurtzite (WZ/WZ), zincblende/zinc-blende (ZB/ZB) and polytype WZ/ZB crystalline phases, as they are currently being synthesized and used in opto-electronic devices. We show that the electronic structure of polytypic CdSe/CdS NCs closely resembles that of WZ or ZB NCs with regard to quantum confinement and strain. This results in similar single-exciton wave functions, with the ( $s$-like) ground state showing quasi-type-II behavior and excited ( $p$-like) states showing fully type-II behavior. The main differences arise in the nature and magnitude of built-in electric fields. We predict these fields are stronger in polytypes than in pure $\mathrm{WZ}$ or ZB NCs due to the sharp spontaneous polarization mismatch between the cubic core and the hexagonal shell lattices. Polarization in NCs is currently believed to be screened by several surface effects. In polytypical structures, however, the polarization mismatch at the interface may create effective charges which are sufficiently far from the outer surface to be quenched. To make a definitive assessment on this controversial issue, we propose experiments in polytypic NCs where both core and shell are made of CdSe, albeit with ZB and WZ structure, respectively. In such a case, band offsets are small, strain is absent and our calculations predict pyroelectricity should become the driving force, inducing transitions from type-I to type-II excitons with increasing core or shell size.
\end{abstract}

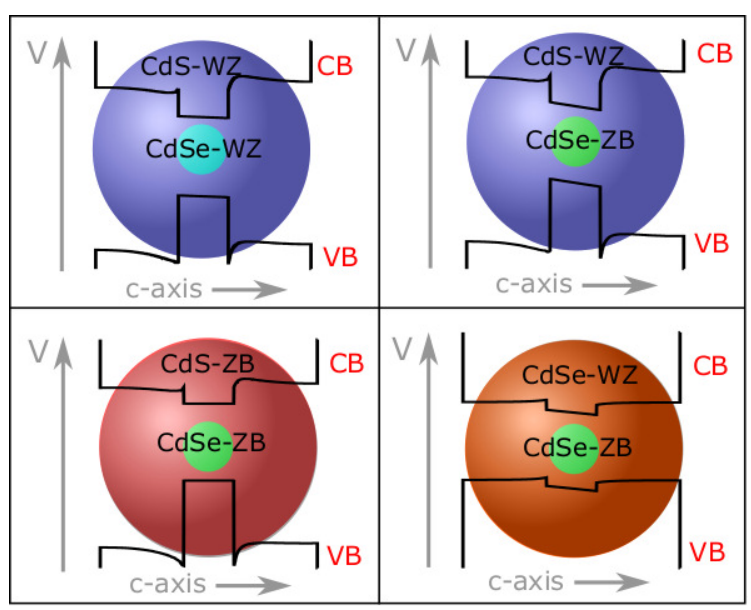




\section{Introduction}

Colloidal NCs with a CdSe core embedded in a thick CdS shell have taken a leading position in the research of semiconductor nanostructures for opto-electronic applications owing to their high luminescence quantum yield, narrow emission linewidth and reduced blinking. ${ }^{1}$ In bulk CdSe and CdS crystals, WZ phase is more stable than ZB one. However, the energy difference is smaller than $1.5 \mathrm{meV} /$ atom. $^{2}$ As a consequence, in NCs -where the surface to volume ratio is high- one can grow either phase through appropriate choice of synthetic temperature or ligands. ${ }^{3-7}$ In the last years, this has made possible the synthesis of CdSe/CdS NCs with pure WZ phase, ${ }^{8-12}$ pure ZB phase ${ }^{5,13-15}$ or a polytypic form where the CdSe core has ZB phase and the CdS shell WZ one. ${ }^{16-18}$

Several spectroscopic studies have analyzed the near-band-edge luminescence of such systems. In all instances, a pronounced dependence of the emission energy on the core and shell size was observed, consistent with a quasi-type-II exciton ground state, where holes are localized inside the core and electrons delocalized over both core and shell. The study of radiative lifetimes, however, reveals quantitative differences between WZ, ZB and ZB/WZ structures. For core radius $\sim 1.5 \mathrm{~nm}$ and thick shells $(\sim 5.5 \mathrm{~nm})$, reported lifetimes in pure WZ NCs range between 100 and $200 \mathrm{~ns},{ }^{19}$ in pure ZB NCs around $50 \mathrm{~ns}^{14}$ and in polytypic NCs around 150 ns. ${ }^{17,20}$ The question arises if such a variation reflects fundamental differences in the electronic structure of the three systems, making excitons in WZ and polytypic NCs longer lived than in ZB ones.

Theoretical studies have used atomistic and continuum models to provide detailed descriptions of the band structure of both $\mathrm{ZB}^{21-23}$ and $\mathrm{WZ}^{19,24} \mathrm{CdSe} / \mathrm{CdS}$ NCs. By contrast, the band structure of polytypic CdSe/CdS NCs remains largely unexplored. Attempts in this direction are essentially limited to simplified effective mass models neglecting strain, perma-

nent dipoles intrinsic to the WZ shell and Coulomb effect on the excitonic wave function. ${ }^{16,17}$ For improved understanding, in the present work we calculate the electronic structure and single exciton states in polytype CdSe/CdS NCs using a multi-band k·p method that ac- 
counts for the above effects. We then compare the results with those of pure WZ and pure ZB heterocrystals, calculated on equal footing, explaining the similarities and analyzing the differences.

Our calculations show that the electronic structure of polytypic NCs resembles that of ZB and WZ NCs. Lattice mismatch strain is comparable in all cases, which results in hydrostatic strain stimulating electron delocalization to a similar extent. The main difference is found in the magnitude and nature of built-in electric fields. These are very weak in ZB NCs because of the cubic lattice symmetry, but reach values of $5-25 \mathrm{mV} / \mathrm{nm}$ inside the core of WZ and polytypic NCs. The fields arise from the competition between piezo and pyroelectricity, the latter being particularly important in polytypic NCs due to the sharp spontaneous polarization mismatch between core and shell lattices. As a result, the core of polytypic NCs -in spite of its ZB phase--experiences stronger fields than those of WZ NCs. The presence of alloyed ZB interlayers, which are often found in polytypic $\mathrm{NCs},{ }^{20}$ barely changes this picture.

Because the origin of internal fields observed in CdSe NCs is a controversial issue, ${ }^{25-31}$ we propose a system which should confirm the presence of spontaneous polarization induced dipoles in core/shell NCs. Namely, polytypic NCs made of CdSe only, albeit with different phase in the core (ZB) and shell (WZ). This turns out to a be strain-free system, hence lacking piezoelectricity, but with sharp polarization mismatch at the core/shell interface. Because band offsets between polytypic forms are low, spontaneous polarization should have a strong influence, and exciton lifetimes should be very sensitive to the core size.

\section{Methods}

The excitonic electron and hole states are calculated with Hamiltonians of the form:

$$
H_{j}=H_{j}^{k i n}+V_{j}+V_{j}^{e-h}
$$


where $j=e, h$ stands for electron or hole, $H_{j}^{k i n}$ is the kinetic energy term, $V_{j}$ the singleparticle confinement potential and $V_{j}^{e-h}$ is Coulomb attraction exerted upon carrier $j$ by the other carrier. The single-particle confinement can be split into several terms:

$$
V_{j}=V_{j}^{\text {conf }}+V_{j}^{s t r}+V_{j}^{p z}+V_{j}^{s p}
$$

where $V_{j}^{\text {conf }}$ the confining potential defined by the band offsets between bulk CdSe and CdS, $V_{j}^{s t r}$ the strain induced deformation potential, $V_{j}^{p z}$ the strain induced piezoelectric potential and $V_{j}^{s p}$ the spontaneous polarization potential.

Strain maps are calculated in the continuous medium model by minimizing the elastic energy. The boundary conditions are zero normal stress for the free surface. ${ }^{32}$ The strain tensor elements, ensuing piezoelectric polarization vector and potential are obtained using the multiphysics mode of Comsol 4.2 software. For electron states, $H_{j}^{k i n}$ is a $3 \mathrm{D}$ singleband effective mass Hamiltonian. For hole states, it is a 3D six-band Hamiltonian for either [111]-grown zinc-blende or wurtzite including $A$-band, $B$-band and $C$-band with spin-orbit interaction, as well as position dependent mass (Burt-Foreman-like). ${ }^{33}$ The strain-induced deformation potential term, $V_{j}^{s t r}$, is isomorphic to the kinetic energy term, ${ }^{34}$ while the piezoelectric and spontaneous polarization potentials, $V_{j}^{p z}$ and $V_{j}^{s p}$, are diagonal. $V_{j}^{e-h}$ is obtained by iterative resolution of the Schrodinger-Poisson equation, within a self-consistent Hartree procedure, taking into account the dielectric mismatch with the dielectric surroundings of the NC. Material parameters are given in the Supporting Information.

\section{Results and discussion}

We compare four different models of $\mathrm{CdSe} / \mathrm{CdS}$ dot-in-dots. The first one is a WZ/WZ core/shell NC with sharp interface, see Fig. 1(a). The second one is a ZB/WZ (polytypic) $\mathrm{NC}$ also with sharp interface, Fig. 1(b). The third one a ZB/WZ NC with a diffusion interlayer in between the core and the shell, Fig. 1(c). The interlayer, which accounts for the 
alloying often observed in polytypic dots, ${ }^{20}$ has ZB structure and CdSe1 $-x \mathrm{~S}_{x}$ composition, with $x$ varying exponentially between the core and shell interfaces. The last model is a ZB/ZB NC, also with sharp interface, Fig. 1(d).

Fig. 1(e-h) shows the corresponding conduction and valence band-edge profiles along the $c$-axis of the WZ phase, which we assume aligned with the [111] ZB direction. The unstrained $\mathrm{CdSe} / \mathrm{CdS}$ band offset potentials (dotted lines) are similar in all cases, forming square wells of similar depth -except for the alloyed NC, Fig. 1(g), where the gradual composition gives rise to a smooth potential barrier-- For the conduction band, the offsets range between 0.32 $\mathrm{eV}$ and $0.43 \mathrm{eV}$.

The band profile changes substantially upon inclusion of strain (dashed lines). Strain deformation potential shifts conduction band and heavy hole band (A-band) edges, and builtin piezoelectric fields are formed due to strain-induced deviations from ideal WZ crystal. As a consequence, the conduction band offset is roughly halved with respect to the unstrained band-offset for all systems. In other words, strain enhances electron delocalization into the shell to a similar extent irrespective of the crystal phase. It is worth noting that this result holds because strain turns out to be very similar regardless of the crystal phase. For pure WZ and pure ZB NCs, the lattice mismatch between CdSe core and CdS shell is known to be $3.9 \%$ and $4.3 \%$, respectively. For the polytypic structure, we find it to be also $\sim 3.9 \%$. After relaxation, the equilibrium strain remains similar in all structures. Even for the alloyed $\mathrm{NC}$, strain in the center of the core is as compressive as that of sharp interface NCs, since it is ultimately set by the thick and stiff CdS shell rather than by the alloyed interlayer (see Supporting Information).

Dashed lines in Fig. 1(f-g) also reveal the presence of significant piezoelectric fields in polytypic NCs. These are very weak in ZB NCs owing to the high lattice symmetry, see Fig. 1(h). In polytypic structures, however, the WZ shell imposes significant fields on the core in spite of its ZB structure. In fact, they reach a magnitude of $13 \mathrm{mV} / \mathrm{cm}$, comparable to that of pure WZ NCs. 


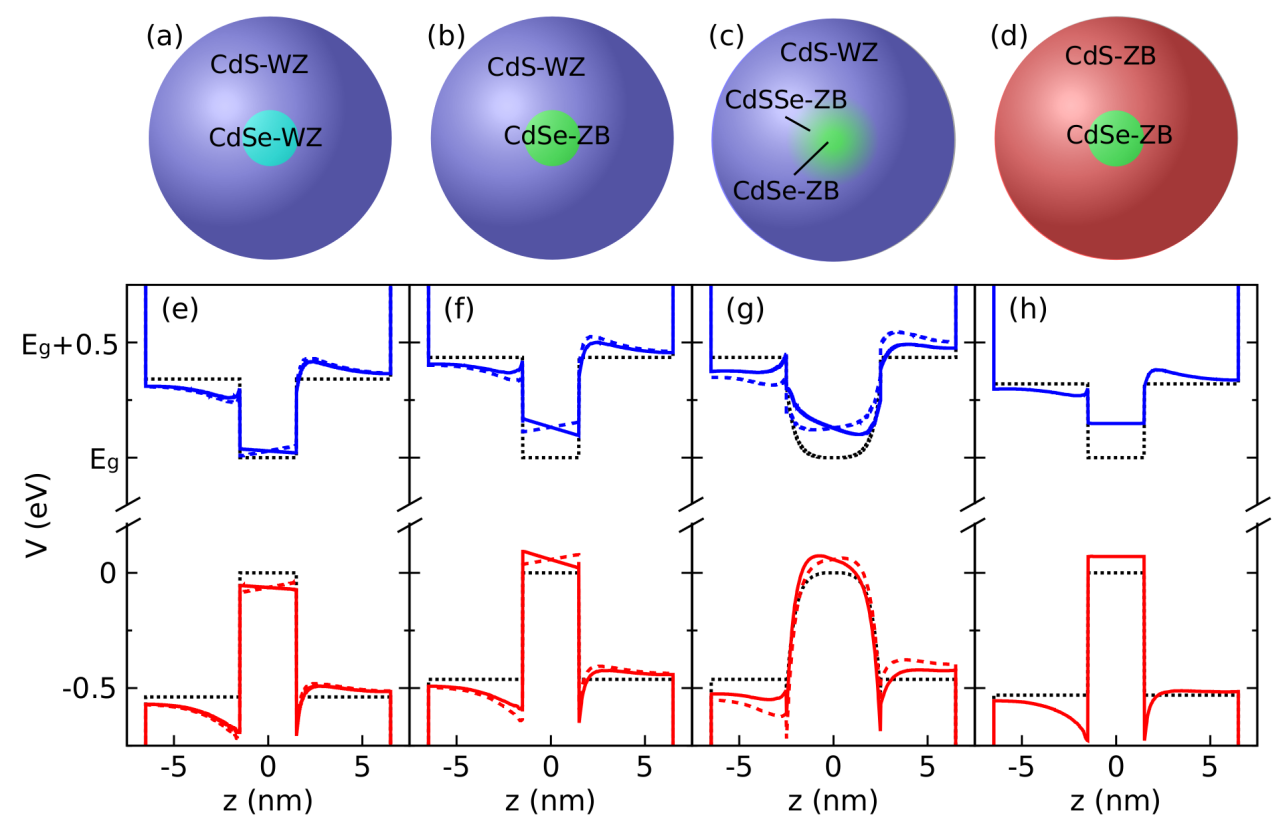

Figure 1: (a-d) Schematic of the CdSe/CdS NCs under study. $R$ is the core radius and $H$ the shell thickness. In (c), we include a 2-nm-thick alloyed interlayer with exponential change of composition between core and shell. (e-h) Corresponding single-particle potential profiles along $c$ axis. Solid lines are used for complete single-particle potential $V_{j}$, dashed lines for potential excluding spontaneous polarization, and dotted ones for unstrained band offset, $V_{j}^{\text {conf }}$. Different colors are used for conduction band (blue) and valence A-band (red). In (e-h), the core has $R=1.5 \mathrm{~nm}$ radius and the shell $H=5 \mathrm{~nm}$.

In addition to piezoelectric fields, one can consider the influence of pyroelectric fields. The latter originate in the spontaneous polarization arising from the intrinsic deviation of WZ crystals from their ideal geometry. This effect had been suggested as the source of permanent dipoles observed in WZ CdSe NCs, ${ }^{25,30}$ although the polarization strength is currently believed to be quenched by several surface effects, including screening by passivating ligands, surface reconstruction and relaxation, ${ }^{27}$ non-stochiometric cation termination of the $\mathrm{NCs}^{28}$ and migration of mobile surface charges. ${ }^{29,31}$ In polytypical structures, however, the polarization mismatch at the heterostructure interface may create effective charges which are sufficiently far from the outer surface to be quenched, as is actually the case of piezoelectric fields. ${ }^{36,37}$ Thus, we next include $V_{j}^{s p}$ in our calculations. We neglect the polarization mismatch at the outer interface, which is presumably quenched, but consider that between 
the core and shell materials. The resulting band profile is represented with solid lines in Fig. 1(e-h).

As can be seen, pyroelectricity compensates and reverses the sign of piezoelectric fields inside the core. For WZ NCs, the field switches from $14 \mathrm{mV} / \mathrm{nm}$ (dashed line) to -6 $\mathrm{mV} / \mathrm{nm}$ (solid line). The opposite sign of pyro and piezoelectric contributions is due to core lattice being compressed. ${ }^{38}$ On the other hand, the magnitude of the pyroelectric field is proportional to the polarization mismatch at the interface. For WZ heterocrystals, $P_{s p}^{C d S e}=$ $-0.42 \mu \mathrm{C} / \mathrm{cm}^{2}$ and $P_{s p}^{C d S}=-0.93 \mu \mathrm{C} / \mathrm{cm}^{2} .{ }^{30}$ For polytypic NCs, in turn, the influence of pyroelectricity is even stronger because the polarization mismatch is maximized, as the ZB core leads to $P_{s p}=0 \mu \mathrm{C} / \mathrm{cm}^{2}$. Consequently, the field felt in the core switches from 13 $\mathrm{mV} / \mathrm{nm}$ (dashed lines) to $-23 \mathrm{mV} / \mathrm{nm}$ (solid lines).

The abovementioned fields are strong enough to open venues for exciton wave function modulation via internal fields, as we recently suggested for pure WZ NCs. ${ }^{19}$ To illustrate this point, in Fig. 2 we plot the squared electron-hole overlap (proportional to radiative lifetimes) for the NCs under study, excluding (a) and including (b) pyroelectric fields. In general, increasing the core size leads to larger overlap, as expected for quasi-type-II systems. ${ }^{35}$ Yet, for large enough core radius $(R>2-2.5 \mathrm{~nm})$ the increasing magnitude of internal dipoles may revert this trend. This can be seen for $\mathrm{WZ} / \mathrm{WZ}$ dots when considering piezoelectric fields only -panel (a)- or for alloyed polytype NCs - panels (a) and (b)-. The exact core size where internal fields overcome quantum confinement effects strongly depends on the conduction band deformation potential parameter, whose value is not precisely determined for hexagonal materials (see Supporting Information). In any case, we stress that this behavior is shared by pure WZ and polytypic NCs, but it is absent in pure ZB NCs where internal fields are negligible. $^{21}$

Altogether, the electron-hole overlaps calculated in Fig. 2 reveal moderate differences between different crystal phases. For $R=1.5 \mathrm{~nm}$ core, we estimate values between 0.4 and 0.5 in all cases. Only for alloyed NCs it is slightly larger (0.6), but this is related to the 
increase in effective volume. We then conclude that the different exciton lifetimes reported in experiments for WZ, ZB and polytype $\mathrm{NCs}^{14,17,19,20}$ are not a consequence of the band structure, but more likely reflect the difficulty of determining exactly the lifetime in NCs with multi-exponential photoluminescence decay. ${ }^{39}$

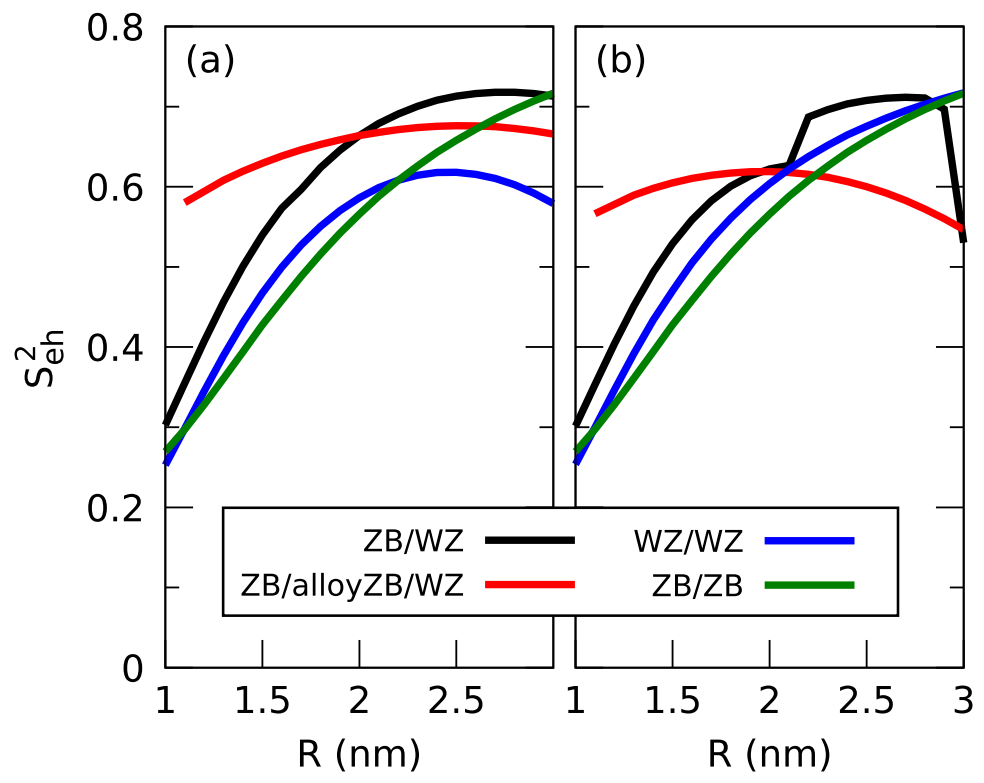

Figure 2: Electron-hole overlap as a function of core size for the four models of CdSe/CdS $\mathrm{NCs}$, (a) excluding and (b) including spontaneous polarization. In all $\mathrm{NCs} H=5 \mathrm{~nm}$. For the alloyed NC, the interlayer is $2 \mathrm{~nm}$ thick. The abrupt changes for ZB/ZB NC in (b) are due to a ground state transition with a nearly degenerate state, taking place for the particular parameters we have chosen. These are not relevant for our analysis.

To investigate the influence of the band profiles of Fig. 1 on the wave functions, in Fig. 3 we compare the electron and hole charge densities for a few low lying single-exciton states in the four prototypical CdSe/CdS NCs we consider. The carrier localization in polytypic structures is similar to that in pure phase structures. In particular, the exciton ground state (left column, s-like orbitals) shows a quasi-type-II behavior, with the hole well localized inside the core and the electron partially leaking into the shell. A similar picture is also found in the case of alloyed interface.

The quasi-type-II behavior and the mild influence of interface alloying on the singleexciton ground state are consistent with photoluminescence experiments in ZB/WZ NCs. ${ }^{18}$ They also agree with, and hence largely validate, simpler effective mass models previously 
used to describe such systems. ${ }^{16,17}$ Note however that such models missed the internal fields arising in polytypic NCs. For typical core sizes, $R=1.5 \mathrm{~nm}$, the potential drop inside the core is still moderate and so is its effect on the carrier localization. Yet, the influence becomes visible in the alloyed NCs, where the smooth confinement facilitates electron-hole charge separation. For larger cores, one should obviously expect an increasingly important role of built-in fields.

While the ground state of CdSe/CdS NCs has been extensively studied by luminiscence techniques, much less is known about excited states, which can be addressed via differential transmission spectra. ${ }^{40}$ To shed light on such states, in the mid and right columns of Fig. 3 we plot the first and second excited states $\left(p_{z}\right.$ and $p_{x} / p_{y}$ orbitals). Interestingly, for all crystal structures the excited states show a clear type-II character, which is markedly different from the ground state. While the hole remains in the core owing to the large band-offset, the electron mostly localizes in the shell, placing its node in the core region. This should render such states optically dark. Note also that $p_{z}$ orbitals are found to be very sensitive to built-in fields in the shell even for ZB NCs.

We have shown so far that the main difference between polytypic and pure phase NCs is the build up of significant pyroelectricity in the core. Still, its effect is generally masked by piezoelectricity and strong quantum confinement. To reduce such competing effects, we next invesigate CdSe-only polytype NCs. It is known that CdSe dots with ZB phase become unstable with increasing size. ${ }^{4,5}$ In what follows we consider a colloidal CdSe dot with ZB phase, which at a certain volume starts growing in WZ phase, thus forming a fully CdSe yet polytypic core/shell NC. The resulting band profile is shown in Fig. 4(a). Neglecting internal fields (dashed line), the band alignment is staggered, with the bottom of the conduction band in the core and the top of the valence band in the shell. Notice however that the band-offsets are small (94 meV for the conduction band, $75 \mathrm{meV}$ for the valence band). The NC is strain free (the lattice constants of CdSe in [111]-grown ZB and in WZ are nearly identical), so that there is no piezoelectricity. Yet, if the crystal growth is ordered, spontaneous polarization 


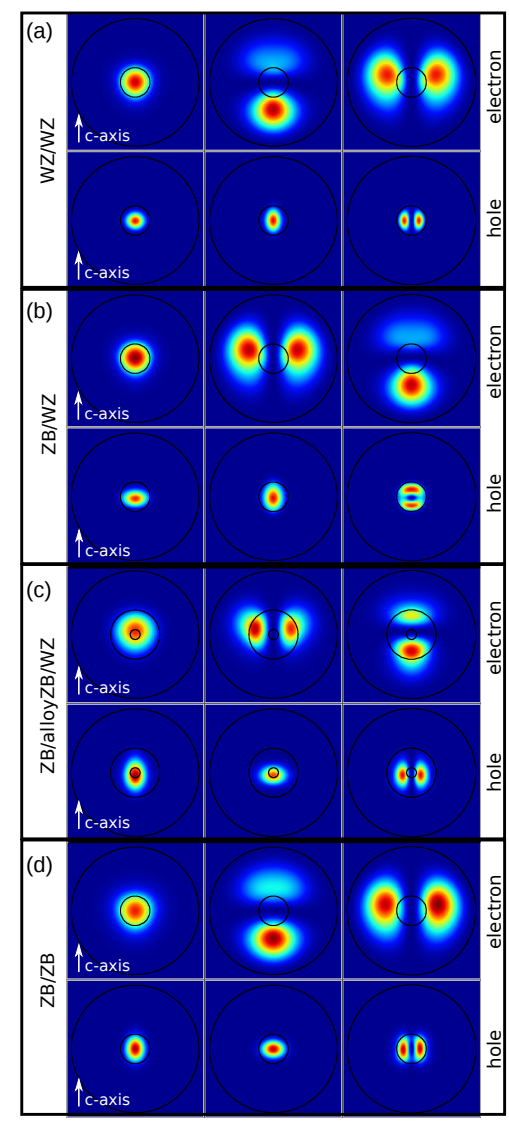

Figure 3: Excitonic electron and hole charge density in the four models of CdSe/CdS NCs we study. (a) WZ/WZ, (b) ZB/WZ, (c) ZB/WZ with alloying and (d) ZB/ZB (bottom panels). The charge densities are similar for polytypic and WZ heterocrystals: quasi-type-II for the ground state and type-II for excited states. In all cases $R=1.5 \mathrm{~nm}$ and $H=5 \mathrm{~nm}$.

should be present. Consequently, internal fields of $\sim 15 \mathrm{mV} / \mathrm{nm}$ build up in the core (solid line).

The band profile described above makes the electronic structure of CdSe polytype NCs very sensitive to the geometry. In Fig. 4(b) we show the excitonic electron and hole charge densities in a NC with thin (left column) and thick (right column) shell. When the shell is thin, electron and hole are largely localized inside the core, because Coulomb interaction and the strong confinement of the shell allow the hole to overcome the valence band offset. By contrast, when the shell becomes thicker the hole moves towards the shell. The effect of varying core radius and shell thickness on the electron-hole overlap is summarized in Fig. 4(c). A qualitative different behavior is observed when including-solid lines-or excluding -dashed 
lines- spontaneous polarization. We thus propose experiments using polytypic CdSe/CdSe NCs to verify the presence of pyroelectric fields in core/shell structures involving WZ phases. A first possibility is to synthesize small core $\mathrm{NCs}(R=1.5 \mathrm{~nm})$ and let the shell thickness increase. If pyroelectricity is present, it should enhance exciton lifetimes, much like in quasitype-II NCs. A second possibility is to keep thin shells $(H=1 \mathrm{~nm})$ and increase the core size. Again, if pyroelectrictiy is present, it should rapidly increase exciton lifetimes.
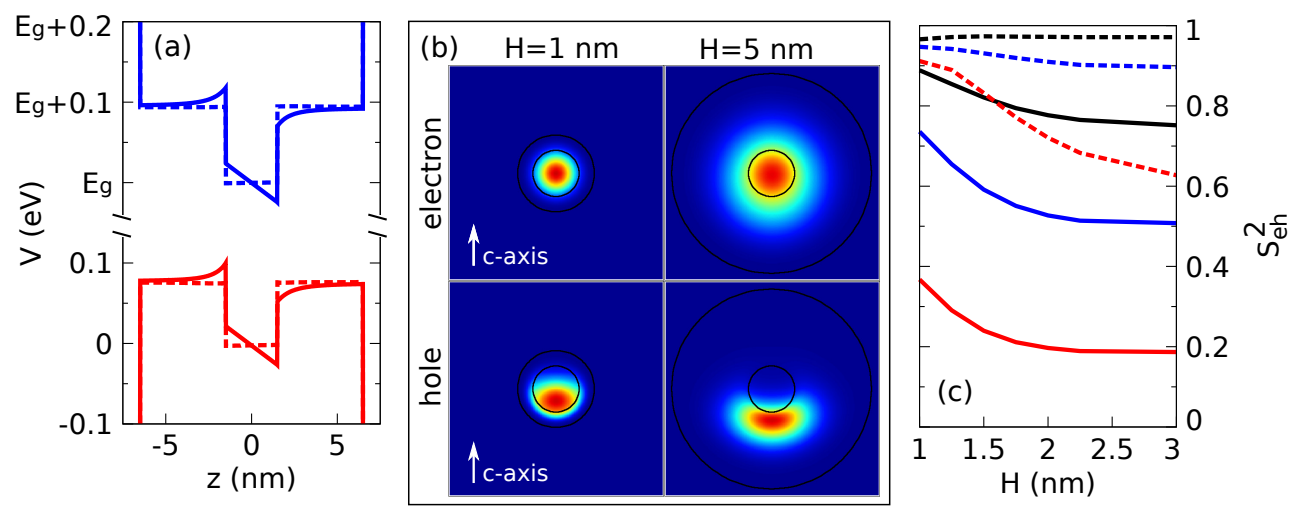

Figure 4: (a) Band profile of polytypic CdSe-only NCs with ZB core and WZ shell. Solid (dashed) lines include (neglect) spontaneous polarization. Notice the type-II band alignment, with built-in electric fields. (b) Exciton's electron (top row) and hole (bottom row) charge densities, for a NC with $R=1.5 \mathrm{~nm}$ and thin (left column) or thick (right column) shell. If the shell is thick, electron and hole evolve towards a type-II exciton. (c) Squared electronhole overlap as a function of NC shell thickness for several radii. Solid (dashed) lines are used for calculations with (without) spontaneous polarization.

Fig. 4 also suggests that polytypic CdSe NCs are potentially interesting structures for tuning carrier-carrier interactions, which is an important capability for several opto-electronic applications. ${ }^{17,35}$ The combination of low band offsets and strong built-in fields allows for an efficient yet controllable exciton lifetime through the core and shell thickness. The polytypic nature may make polarization effects more robust than in WZ CdSe dots. On the other hand, as compared to CdSe/CdS heterostructures, the low band offsets of CdSe-only polytypes should imply weaker Auger recombination, ${ }^{41,42}$ which is convenient for several optical applications. 


\section{Conclusions}

We have studied the influence of polytypism in ZB/WZ core/shell NCs using $\mathrm{k} \cdot \mathrm{p}$ theory. In CdSe/CdS heterocrystals, we find quantum confinement and strain are comparable to those of pure WZ and pure ZB NCs. Internal fields are formed inside the ZB core due to piezoelectricity and pyroelectricity imposed by the WZ shell. In this regard, polytypic structures resemble WZ NCs rather than ZB ones. Interface alloying softens the confinement potential, but leaves strain and built-in fields essentially unaltered. As a result, low-energy excitons of polytypic NCs have quantitatively similar properties to their WZ counterparts, which is consistent with experiments. The main difference is the strength of pyroelectricity, which we predict to be stronger than in WZ heterocrystals.

To test unambiguously the presence of pyroelectricity in core/shell structures, we propose the study of polytypical CdSe/CdSe NCs. These are strain-free objects with low band offsets. In these conditions, pyroelectricity emerges as a leading factor determining the electronic structure. Should experiments confirm our predictions, polytypic CdSe/CdSe NCs -with high sensitivity of the exciton wave function to core and shell dimensions- could become advantageous structures for opto-electronic applications where tuning of carrier-carrier interactions is required, such as lasing, non-linear optics, photodetectors and photovoltaic cells. $^{35}$

\section{Acknowledgement}

We thank I. Moreels and P. Guyot-Sionnest for useful discussions. Support from MINECO project CTQ2014-60178-P, UJI project P1-1B2014-24 is acknowledged. 


\section{References}

(1) Kovalenko M.; Manna L.; Cabot A.; Hens Z.; Talapin D.V.; Kagan Ch.R.; Klimov V.I.; Rogach A.L.; Reiss P.; Milliron D.J.; et al. Prospects of Nanoscience with Nanocrystals. ACS Nano 2015, 9, 1012-1057.

(2) Yeh C.Y.; Lu Z.W.; Zunger A. Zinc-blende - Wurtzite Polytypism in Semiconductors. Phys. Rev. B 1992, 46, 10086-10097.

(3) Huang J.; Kovalenko M.V.; Talapin D.V. Alkyl Chains of Surface Ligands Affect Polytypism of CdSe Nanocrystals and Play an Important Role in the Syntheis of Anisotropic Nanoheterostructures. J. Am. Chem. Soc. 2010, 132, 15866-15868.

(4) Nag A.; Hazarika A.; Shanavas K.V.; Sharma S.M.; Dasgupta I.; Sarma D.D. Crystal Structure Engineering by Fine-Tuning the Surface Energy: The Case of CdE (E=S/Se) Nanocrystals. J. Phys. Chem. Lett. 2011, 2, 706-712.

(5) Mahler B.; Lequeux N.; Dubertret B. Ligand-Controlled Polytypism of Thick-Shell CdSe/CdS Nanocrystals. J. Am. Chem. Soc. 2010, 132, 953-959.

(6) Gao Y.; Peng X. Crystal Structure Control of CdSe Nanocrystals in Growth and Nucleation: Dominating Effects of Surface versus Interior Structure. J. Am. Chem. Soc. 2014, 136, 6724-6732.

(7) Majumder S.; Bae I.T.; Maye M.M. Investigating the Role of Polytypism in the Growth of Multi-Shell CdSe/CdZnS Quantum Dots. J. Mater. Chem. C 2014, 2, 4659.

(8) Mahler B.; Spinicelli P.; Buil S.; Quelin X.; Hermier J.P.; Dubertret B. Towards NonBlinking Colloidal Quantum Dots. Nat. Mater. 2008, 7 659-664.

(9) Chen O.; Zhao J.; Chauhan V.P.; Cui J.; Wong C.; Harris D.K.; Wei H.; Han H.S.; Fukumura D.; Jain R.K.; Bawendi M.G. Compact High-Quality CdSe/CdS Core/Shell 
Nanocrystals with Narrow Emission Linewidths and Suppressed Blinking. Nat. Mater. 2013, 12, 445-451.

(10) Cirillo M.; Aubert T.; Gomes R.; Deun R.V.; Emplit P.; Biermann A.; Lange H.; Thomsen C.; Brainis E.; Hens Z. "Flash" Synthesis of CdSe/CdS CoreShell Quantum Dots. Chem. Mater. 2014, 26, 1154-1160.

(11) Christodoulou S.; Vaccaro G.; Pinchetti V.; De Donato F.; Grim J.Q.; Casu A.; Genovese A.; Vicidomini G.; Diaspro A.; Brovelli S.; et al. Synthesis of Highly Luminescent Wurtzite CdSe/CdS Giant-shell Nanocrystals Using a Fast Continuous Injection Route. J. Mater. Chem. C 2014, 2, 3439-3447.

(12) van Embden J.; Jaseniak J.; Mulvaney P. Mapping the Optical Properties of CdSe/CdS Heterostructure Nanocrystals: The Effects of Core Size and Shell Thickness. J. Am. Chem. Soc. 2009, 131, 14299-14309.

(13) Nan W.; Niu Y.; Qin H.; Cui F.; Yang Y.; Lai R.; Lin W.; Peng X. Crystal Structure Control of Zinc-Blende CdSe/CdS Core/Shell Nanocrystals: Synthesis and StructureDependent Optical Properties J. Am. Chem. Soc. 2012, 134, 19685-19693.

(14) Qin H.; Niu Y.; Meng R.; Lin X.; Lai R.; Fang W.; Peng X. Single-Dot Spectroscopy of Zinc-Blende CdSe/CdS Core/Shell Nanocrystals: Nonblinking and Correlation with Ensemble Measurements. J. Am. Chem. Soc. 2014, 136, 179-187.

(15) Gong K.; Martin J.E.; Shea-Rohwer L.E.; Lu P.; Kelley D.F. Radiative Lifetimes of Zincblende CdSe/CdS Quantum Dots. J. Phys. Chem. C 2015, 119, 2231-2238.

(16) Garcia-Santamaria F.; Chen Y.; Vela J.; Schaller R.D.; Hollingsworth J.A.; Klimov V.I. "Giant" Nanocrystals Boosts Optical Gain Performance. Nano Lett. 2009, 9, 34823488. 
(17) Brovelli S.; Schaller R.D.; Crooker S.A.; Garcia-Santamaria F.; Chen Y.; Viswanatha R.; Hollingsworth J.A.; Htoon H.; Klimov V.I. Nano-engineered electron-hole exchange interaction controls exciton dynamics in core-shell semiconductor nanocrystals. Nat. Commun. 2011, 2, 280.

(18) Bae W.K.; Padilha L.A.; Park Y.S.; McDaniel H.; Robel I.; Pietryga J.M.; Klimov V.I. Controlled Alloying of the Core-Shell Interface in CdSe/CdS Quantum Dots for Suppression of Auger Recombination. ACS Nano 2013, 7, 3411-3419.

(19) Segarra C.; Climente J.I.; Polovitsyn A.; Rajadell F.; Moreels I.; Planelles J. Piezoelectric Control of the Exciton Wave Function in Colloidal CdSe/CdS Nanocrystals. J. Phys. Chem. Lett. 2016, 7, 2182-2188.

(20) Pinchetti V.; Meinardi F.; Camellini A.; Sirigu G.; Christodoulou S.; Bae W.K.; De Donato F.; Manna L.; Zavelani-Rossi M. et al. Effect of Core/Shell Interface on Carrier Dynamics and Optical Gain Properties of Dual-Color Emitting CdSe/CdS Nanocrystals. ACS Nano 2016, 10, 6877-6887.

(21) Kocevski V.; Rusz J.; Eriksson O.; Sarma D.D. First-Principles Study of the Influence of Different Interfaces and Core Types on the Properties of CdSe/CdS Core-Shell Nanocrystals. Sci. Rep. 2015, 5, 10865.

(22) Tschirner N.; Lange H.; Schliwa A.; Biermann A.; Thomsen C.; Lambert K.; Gomes R.; Hens Z. Interfacial Alloying in CdSe/CdS Heteronanocrystals: A Raman Spectroscopy Analysis. Chem. Mater. 2011, 24, 311-318.

(23) Dong L.; Sugunan A.; Hu J.; Zhou S.; Li S.; Popov S.; Toprak M.S.; Friberg A.T.; Muhammed M. Photoluminescence from Quasi-Type-II Spherical CdSe-CdS Core-Shell Quantum Dots. Appl. Opt. 2013, 52, 105-109.

(24) Park S.H.; Cho Y.H. Strain and Piezoelectric Potential Effects on Optical Properties in CdSe/CdS Core/Shell Quantum Dots. J. Appl. Phys. 2011, 109, 113103. 
(25) Blanton S.A.; Leheny R.L.; Hines M.A.; Guyot-Sionnest P. Dielectric Dispersion Measurements of CdSe Nanocrystal Colloids: Observation of a Permanent Dipole Moment. Phys. Rev. Lett. 1997, 79, 865-868.

(26) Li L.S.; Alivisatos A.P. Origin and Scaling of the Permanent Dipole Moment in CdSe Nanorods. Phys. Rev. Lett. 2003, 90, 097402.

(27) Rabani E. Structure and electrosatic properties of passivated CdSe nanocrystals. J. Chem. Phys. 2001, 115, 1493-1497.

(28) Taylor J.; Kippeny T.; Bennett J.C.; Huang, M.: Feldman L.C.; Rosenthal S.J. Surface Stoichiometry of CdSe Nanocrystals, MRS Proceedings 1998, doi: 10.1557/PROC-536413.

(29) Müller J.; Lupton J.M.; Rogach A.L.; Feldmann J. Monitoring Surface Charge Migration in the Spectral Dynamics of Single CdSe/CdS Nanodot/Nanorod Heterostructures. Phys. Rev. B 2005, 72, 205339.

(30) Nann T.; Schneider J. Origin of Permanent Electric Dipole Moments in Wurtzite Nanocrystals. Chem. Phys. Lett. 2004, 384 150-152.

(31) Shim M.; Guyot-Sionnest P. Permanent Dipole Moment and Charges in Colloidal Semiconductor Quantum Dots. J. Chem. Phys. 1999, 111, 6955-6964.

(32) Rajadell F.; Royo M.; Planelles J. Strain in Free Standing CdSe/CdS Core-Shell Nanorods. J. Appl. Phys. 2012, 111, 014303.

(33) Climente J.I.; Segarra C.; Rajadell F.; Planelles J. Electrons, Holes, and Excitons in GaAs Polytype Quantum Dots. J. Appl. Phys. 2016, 119, 125705.

(34) Chuang S.L.; Chang C.S. k·p Method for Strained Wurtzite Semiconductors. Phys. Rev. $B$ 1996, 54, 2491-2503. 
(35) Piryatinski A.; Ivanov S.A.; Tretiak S.; Klimov V.I. Effect of Quantum and Dielectric Confinement on the Exciton-Exciton Interaction Energy in Type-II Core/Shell Semiconductor Nanocrystals. Nano Lett. 2007, 7, 108-115.

(36) Morello G.; Della Sala F.; Carbone L.; Manna L.; Maruccio G.; Cingolani R.; De Giorgi M. Intrinsic Optical Nonlinearity in Colloidal Seeded Grown CdSe/CdS Nanostructures: Photoinduced Screening of the Internal Electric Field. Phys. Rev. B 2008, 78, 195313.

(37) Christodoulou S.; Rajadell F.; Casu A.; Vaccaro G.; Grim J.; Genovese A.; Manna L.; Climente J.I.; Meinardi F.; Raino G.; et al. Band Structure Engineering via Piezoelectric Fields in Strained Anisotropic CdSe/CdS Nanocrystals. Nat. Commun. 2015, 6, 7905.

(38) Schliwa A.; Hönig G.; Bimberg D. in Multi-Band Effective Mass Approximations, M. Ehrhardt and T. Koprucki (Eds), Springer, Heidelberg, 2014.

(39) Gong K.; Zeng Y.; Kelley D.F. Exctinction Coefficients, Oscillator Strengths, and Radiative Lifetimes of CdSe, CdTe, and CdTe/CdSe Nanocrystals. J. Phys. Chem. C 2013, 117, 20268-20279.

(40) Lupo M.G.; Scotognella F.; Zavelani-ROssi M.; Lanzani G.; Manna L.; Tassone F. Band-edge ultrafast pump-probe spectroscopy of core/shell CdSe/CdS rods: assesing electron delocalization by effective mass calculations. Chem. Phys. Phys. Chem. 2012, 14, 7420-7426.

(41) Cragg, G. E.; Efros, A. L. Suppression of Auger Processes in Confined Structures. Nano Lett. 2010 10, 313-317.

(42) Climente J.I.; Movilla J.L.; Planelles J. Auger Recombination Suppression in Nanocrystals with Asymmetric Electron-Hole Confinement. Small 2012, 8, 754-759. 